\title{
Prophylaxis of venous thromboembolism in medical patients: too much or too little?
}

This article was published in the following Dove Press journal:

Clinical Epidemiology

14 November 2012

Number of times this article has been viewed

\section{Christian Fynbo \\ Christiansen}

Department of Clinical Epidemiology, Aarhus University Hospital, Aarhus, Denmark
Correspondence: Christian Fynbo Christiansen

Department of Clinical Epidemiology, Aarhus University Hospital, Olof Palmes Alle 43-45, DK-8200 Aarhus C, Denmark Tel +45 87| 682 I8

Fax +45 87I 672 I5

Email cc@dce.au.dk

\begin{abstract}
Venous thromboembolism (VTE) is a potentially serious complication occurring in $1 \%-2 \%$ of hospitalized medical patients. Despite this low absolute risk, as many as $82 \%$ of medical patients are considered to be at increased risk of developing VTE and are eligible for medical thromboprophylaxis. In this commentary, The author will discuss the main findings of a recent paper published in Clinical Epidemiology that questions the large proportion of individuals who are eligible for medical thromboprophylaxis, and also discuss the potential implications for the prevention of VTE. The recent paper demonstrated that when a population is divided into high- and low-risk groups, the maximum absolute risk depends on the inverse of the proportion of patients that is considered to be high risk. Consequently, even an effective treatment will only result in a small reduction in the absolute risk when the high-risk group comprises the largest proportion of this population. For medical thromboprophylaxis, this implies that even patients considered to be at high-risk for developing VTE have a maximum absolute VTE risk of $2 \%$ when the overall risk is $1.6 \%$. Therefore, even an effective preventive initiative will only result in a small risk reduction. This small potential benefit should be weighed against potential harms associated with prophylaxis, mainly bleeding events. Still, there may be a reasonable overall balance between prevention of pulmonary embolism and major bleeding, mainly because major bleeding events are rare. Nonetheless, this discussion underscores that future risk prediction models should aim to predict the benefits and harms in individual patients in order to provide optimal care for the right patients.
\end{abstract}

Keywords: comment, epidemiology, prevention, risk factors, venous thromboembolism

\section{Venous thromboembolism in medical patients}

Venous thromboembolisms (VTE), including deep venous thrombosis (DVT) and pulmonary embolism (PE), are potentially serious complications in hospitalized medical patients. Risk factors for the development of VTE in medical patients include (among others) increased age, intensive care admission, hormone replacement therapy, cancer, and other comorbidities including cardiovascular, renal, and respiratory diseases. ${ }^{1}$

According to US and UK guidelines, medical patients with reduced mobility and the presence of one or more risk factors are considered to be at risk for developing VTE, and are thus eligible for medical thromboprophylaxis unless they have contraindications leaving them prone to bleeding events. ${ }^{2,3}$ As many as $82 \%$ of medical patients have at least one risk factor and are thus eligible for medical thromboprophylaxis. ${ }^{4,5}$ Still, only half of eligible medical patients receive thromboprophylaxis despite several initiatives to improve adherence to the guidelines. ${ }^{4,6,7}$

The question is thus: is it reasonable to provide medical thromboprophylaxis to the majority of medical patients, or would this result in overtreatment, inducing more 
harm than benefit? The answer to this question is not easy and ultimately depends on our knowledge and assumptions about the risk of VTE and the beneficial and adverse effects of medical thromboprophylaxis.

In this commentary, the author will briefly discuss a recent paper by Millar, ${ }^{8}$ which challenges the definition of a highrisk group, discusses the assumptions underlying the ongoing discussion about thromboprophylaxis in medical patients, and touches upon potential areas for future research.

\section{Risk ofVTE in medical patients}

The risk of VTE can be defined as the probability of a VTE event occurring during a specified period of time. The comparison of studies assessing this is hampered by the varying follow-up times used to determine the onset of VTE, with some studies using fixed time periods, while others include only VTE occurring during hospitalization, which depends on the length of hospital stay.

A meta-analysis from 2011 that included clinical trials measuring clinical outcomes up to 120 days after medical admission found a $0.96 \%$ risk of DVT and a $1.2 \%$ risk of PE when control groups were pooled. ${ }^{9}$ In a US study by Edelsberg et $\mathrm{al}^{10}$ that included hospitalized medical patients aged 40 years or above, the 90 -day risk of being registered with a diagnosis of VTE was $1.59 \%$, of which $18 \%$ occurred after hospital discharge. In contrast, the risk of asymptomatic DVT may be as high as $24 \% .^{11}$

\section{Medical thromboprophylaxis}

The meta-analysis from 2011 included 21 randomized trials that followed medical patients for up to 120 days, to assess clinical outcomes. ${ }^{9}$ The study found that medical thromboprophylaxis with heparins was associated with a reduction in PE risk from $1.1 \%$ to $0.82 \%$ (odds ratio [OR] $=0.70 ; 95 \%$ CI: 0.56-0.87), while the effect was less clear on symptomatic DVT $(0.97 \%$ versus $0.77 \%$ [OR $=0.75 ; 0.43-1.30]$ ) and mortality $(8.2 \%$ versus $7.5 \%[\mathrm{OR}=0.93 ; 0.86-1.00])$. Treatment was, however, associated with an increased risk of any bleeding events $(3.2 \%$ versus $5.0 \%[\mathrm{OR}=1.28 ; 95 \% \mathrm{CI}$ : $1.05-1.56])$ including major bleeding events $(0.58 \%$ versus $0.77 \%$ [OR $=1.61 ; 95 \% \mathrm{CI}: 1.23-2.10]) .{ }^{9}$

The findings from the meta-analyses were recently confirmed by the LIFENOX trial, which randomized more than 8000 acutely ill medical patients to low-molecular weight heparin (enoxaparin) in addition to elastic stockings. ${ }^{12}$ The study found no impact on overall 14-, 30-, and 90-day mortality. There were no increased rates of clinical PE or cardiovascular death, but the rate of bleeding was higher in the enoxaparin groups compared with the placebo group ( $2.2 \%$ versus $1.5 \%$, respectively), while there were virtually no difference in major bleedings $(0.4 \%$ versus $0.3 \%$, respectively).

While the meta-analysis and the LIFENOX trial focused on clinical outcomes, previous trials on asymptomatic VTE reached other conclusions. For example, in the MEDENOX trial, which included 1102 medical patients, enoxaparin reduced the frequency of asymptomatic VTE to 5.5\% compared with $14.9 \%$ in the placebo group, while there was no effect on mortality. ${ }^{13}$ Patients were systematically examined for DVT by venography or venous ultrasonography. This illustrates that the definition of VTE has a major impact on the findings, and thereby on the data used to assess the effect of thromboprophylaxis as discussed below.

\section{Defining high-risk for venous thromboembolism: theoretical and clinical implications}

The recent paper by Millar ${ }^{8}$ addresses the challenges when large proportions of individuals are considered to be at risk for a certain outcome. The paper mathematically proves that when a population is divided dichotomously into high- and low-risk groups, the maximum absolute risk and incremental risk in the high-risk group depends on or equals the inverse of the proportion of a population that is considered to be high risk. The results are striking when applied to the prevention of VTE. While as many as $82 \%$ of medical patients are considered to be at high risk for VTE, ${ }^{4}$ and the overall risk of VTE is assumed to be $1.59 \%,{ }^{10}$ the maximum risk in the high-risk group cannot exceed $1.94 \%$, even if the risk level in the low-risk group was $0.00 \%{ }^{8}$ With such a low absolute risk in the high-risk group, even a very effective treatment would only lead to a small absolute risk reduction. When the high-risk group is large, the low-risk group becomes small, and is not representative of the entire population. The study by Millar ${ }^{8}$ also highlights that relative risk is a poor measure in these situations.

However, the study conclusions rely on several assumptions. The model presented in the study simply summed the proportions of patients with each risk factor and did not take into account the co-occurrence of more than one risk factor in each patient, although there are usually several factors that interact to cause disease. ${ }^{14}$ This may not influence the main conclusion, but it highlights the fact that intermediate results should be interpreted cautiously. Consequently, single risk factors cannot simply be removed from the model. Importantly, the study assumed that $82 \%-88 \%$ of medical 
patients have a risk factor for VTE, while this rate may only be $41 \%$ according to US guidelines. ${ }^{5}$ In addition, the conclusion relies on the assumption that VTE risk was based on the US study, which includes diagnoses registered within 90 days after medical admission. ${ }^{10}$ This is a reasonable estimate, as the meta-analyses found a $1 \%$ approximate risk that patients are symptomatic for both DVT and PE. ${ }^{9,15}$ However, screening with venography or ultrasound may reveal asymptomatic VTE in at least tenfold more patients. ${ }^{11}$ The decision about providing medical thromboprophylaxis thus depends on which outcome should be prevented. The ultimate goal must be to improve patient prognosis with regard to mortality and morbidity, including postthrombotic complications. These complications include postthrombotic syndrome (which occurs in more than $20 \%$ of patients with symptomatic DVT after 2 years, and can also increase to more than $50 \%$ within 10 years), ${ }^{16,17}$ as well as chronic thromboembolic pulmonary hypertension occurring in 3\% of patients treated for PE. ${ }^{18}$ Whether asymptomatic VTE in medical patients is associated with mortality and morbidity is less clear.

\section{Potential clinical implications}

The paper by Millar ${ }^{8}$ may have clinical implications by highlighting the fact that VTE is an infrequent event, even in patients considered to be at high risk for developing VTE. The clinical decision to initiate an intervention in patients relies on balancing benefits and harms. In other words, for a given intervention, the number needed to treat should be lower than the number needed to harm. Computing these numbers ultimately depends on valid data pertaining to the risk of the outcome, the efficacy of the intervention, and the safety of the intervention. There are, however, conflicting data about these figures in VTE, which may explain much of the ongoing discussions.

The 2011 meta-analysis may provide data for such a comparison between the benefits associated with the prevention of PE and the harms related to major bleedings. ${ }^{9}$ The risk of $\mathrm{PE}$ in patients with stroke and other medical conditions was $0.82 \%$ after receiving medical prophylaxis, and $1.1 \%$ in the control groups; therefore, the risk difference was $0.28 \%$. This corresponds to a number needed to treat (the inverse of the risk reduction) of 352; in other words, 352 medical patients must be treated in order to prevent one PE event. The risk of major bleeding was $0.77 \%$ in treated patients and $0.58 \%$ in the control groups; therefore, the risk difference was $0.21 \%$. This corresponds to a number needed to harm (the inverse of the attributable risk) of 485 . With these figures, treatment benefits outweigh harms despite the low risk of VTE. There may, however, be subsets of patients or other outcomes in which there is imbalance between benefits and harms. In addition, patients included in trials may not be representative of all medical patients, thereby limiting generalizability. ${ }^{19}$

\section{Perspectives}

How could we improve the balance between benefits and harms? First, we must improve our knowledge of the number needed to treat and the number needed to harm. We therefore need valid real-world data on the risk ofVTE, and the effects and adverse events of medical thromboprophylaxis in medical patients. Such data could be provided by observational studies, because trials often include selected populations. ${ }^{19}$ Second, we should have even more data pertaining to benefits versus harms within subsets of medical patients in order to identify individual patients who will benefit from medical thromboprophylaxis. Third, with the increasing use of information technology in the health care system, more detailed risk prediction models could be developed, validated, and implemented in electronic medical records. ${ }^{20-22}$ As an example, such a prediction model has been developed for estimating the long-term risk of VTE in general practice. ${ }^{23}$

\section{Conclusion}

In conclusion, current guidelines advocate for VTE prophylaxis among most medical patients, despite the fact that they only have a $1 \%-2 \%$ risk of developing VTE. This may be reasonable given that harms, primarily major bleeding events, are even rarer. Future risk prediction models should aim to predict the benefits and harms among individual patients in order to provide the optimal care at an individual level.

\section{Disclosure}

The author reports no conflicts of interest in this work.

\section{References}

1. Rocha AT, Paiva EF, Lichtenstein A, Milani R Jr, Cavalheiro CF, Maffei FH. Risk-assessment algorithm and recommendations for venous thromboembolism prophylaxis in medical patients. Vasc Health Risk Manag. 2007;3(4):533-553.

2. Hill J, Treasure T; for National Clinical Guideline Centre for Acute and Chronic Conditions. Reducing the risk of venous thromboembolism in patients admitted to hospital: summary of NICE guidance. BMJ. 2010;340:c95.

3. Geerts WH, Bergqvist D, Pineo GF, et al; for American College of Chest Physicians. Prevention of venous thromboembolism: American College of Chest Physicians Evidence-Based Clinical Practice Guidelines (8th Edition). Chest. 2008;133(Suppl 6):381S-453S.

4. Gibbs H, Fletcher J, Blombery P, Collins R, Wheatley D. Venous thromboembolism prophylaxis guideline implementation is improved by nurse directed feedback and audit. Thromb J. 2011;9(1):7. 
5. Cohen AT, Tapson VF, Bergmann JF, et al; for ENDORSE Investigators. Venous thromboembolism risk and prophylaxis in the acute hospital care setting (ENDORSE study): a multinational cross-sectional study. Lancet. 2008;371(9610):387-394.

6. Herzig SJ, Rothberg MB. Prophylaxis rates for venous thromboembolism and gastrointestinal bleeding in general medical patients: too low or too high? BMJ. 2012;344:e3248.

7. Anderson FA Jr, Goldhaber SZ, Tapson VF, et al; for ENDORSE Investigators. Improving Practices in US Hospitals to Prevent Venous Thromboembolism: lessons from ENDORSE. Am J Med. 2010; 123(12):1099-1106. e8

8. Millar JA. Measures of risk and their relationship to the relative size of the high-risk group: application to medical thromboprophylaxis. Clin Epidemiol. 2012;4:275-281.

9. Lederle FA, Zylla D, MacDonald R, Wilt TJ. Venous thromboembolism prophylaxis in hospitalized medical patients and those with stroke: a background review for an American College of Physicians Clinical Practice Guideline. Ann Intern Med. 2011;155(9):602-615.

10. Edelsberg J, Hagiwara M, Taneja C, Oster G. Risk of venous thromboembolism among hospitalized medically ill patients. Am J Health Syst Pharm. 2006;63(20 Suppl 6):S16-S22.

11. Venous Thromboembolism: Reducing the Risk of Venous Thromboembolism (Deep Vein Thrombosis and Pulmonary Embolism) in Patients Admitted to Hospital. London, UK: National Clinical Guideline Centre - Acute and Chronic Conditions; 2010. Available from: http:// www.nice.org.uk/nicemedia/live/12695/47920/47920.pdf. Accessed November 7, 2012.

12. Kakkar AK, Cimminiello C, Goldhaber SZ, Parakh R, Wang C, Bergmann JF; for LIFENOX Investigators. Low-molecular-weight heparin and mortality in acutely ill medical patients. $N$ Engl J Med. 2011;365(26):2463-2472.

13. Samama MM, Cohen AT, Darmon JY, et al. A comparison of enoxaparin with placebo for the prevention of venous thromboembolism in acutely ill medical patients. Prophylaxis in Medical Patients with Enoxaparin Study Group. N Engl J Med. 1999;341(11):793-800.
14. Rothman KJ, Greenland S, Poole C, Lash TL. Causation and causal inference. In: Rothman KJ, Greenland S, Lash TL, editors. Modern Epidemiology. 3rd ed. Philadelphia: Lippincott William \& Wilkins; 2008:5-31.

15. Kahn SR, Lim W, Dunn AS, et al; for American College of Chest Physicians. Prevention of VTE in nonsurgical patients: Antithrombotic Therapy and Prevention of Thrombosis, 9th ed: American College of Chest Physicians Evidence-Based Clinical Practice Guidelines. Chest. 2012;141(Suppl 2):e195S-e226S

16. Prandoni P, Lensing AW, Cogo A, et al. The long-term clinical course of acute deep venous thrombosis. Ann Intern Med. 1996;125(1):1-7.

17. Schulman S, Lindmarker P, Holmstrom M, et al. Post-thrombotic syndrome, recurrence, and death 10 years after the first episode of venous thromboembolism treated with warfarin for 6 weeks or 6 months. J Thromb Haemost. 2006;4(4):734-742.

18. Kearon C, Akl EA, Comerota AJ, et al; for American College of Chest Physicians. Antithrombotic therapy for VTE disease: Antithrombotic Therapy and Prevention of Thrombosis, 9th ed: American College of Chest Physicians Evidence-Based Clinical Practice Guidelines. Chest. 2012;141(Suppl 2):e419S-e494S

19. Sørensen HT, Lash TL, Rothman KJ. Beyond randomized controlled trials: a critical comparison of trials with nonrandomized studies. Hepatology. 2006;44(5):1075-1082.

20. Prandoni P, Samama MM. Risk stratification and venous thromboprophylaxis in hospitalized medical and cancer patients. Br J Haematol. 2008;141(5):587-597.

21. Kucher N, Koo S, Quiroz R, et al. Electronic alerts to prevent venous thromboembolism among hospitalized patients. $N$ Engl J Med. 2005;352(10):969-977.

22. Piazza G, Goldhaber SZ. Computerized decision support for the cardiovascular clinician: applications for venous thromboembolism prevention and beyond. Circulation. 2009;120(12):1133-1137.

23. Hippisley-Cox J, Coupland C. Development and validation of risk prediction algorithm (QThrombosis) to estimate future risk of venous thromboembolism: prospective cohort study. BMJ. 2011;343:d4656.
Clinical Epidemiology

\section{Publish your work in this journal}

Clinical Epidemiology is an international, peer-reviewed, open access journal focusing on disease and drug epidemiology, identification of risk factors and screening procedures to develop optimal preventative initiatives and programs. Specific topics include: diagnosis, prognosis, treatment, screening, prevention, risk factor modification, systematic

\section{Dovepress}

reviews, risk \& safety of medical interventions, epidemiology \& biostatical methods, evaluation of guidelines, translational medicine, health policies \& economic evaluations. The manuscript management system is completely online and includes a very quick and fair peer-review system, which is all easy to use. 